\author{
Izabela Gołębiowska \\ University of Warsaw \\ Faculty of Geography and Regional Studies \\ Department of Cartography \\ e-mail:i.michna@uw.edu.pl
}

\title{
THE ROLE OF MAP LEGENDS \\ IN THE PROCESS OF MAP USE. CONCLUSIONS FROM AN EXPLORATORY STUDY
}

\begin{abstract}
Map and geospatial information is an essential tool in many fields of human activities. Although cartographers were extensively involved in cognitive map design research during the second part of the $20^{\text {th }}$ century, the map use process as well as the significance of individual map design elements have not been studied in sufficient depth. The article discusses the results of a controlled experiment. The exploratory study aimed to simulate the execution of some problem-solving tasks based on the information derived from thematic maps with differently designed legends, viz.: list-legend, grouping-legend and natural-legend. On the basis of think-aloud protocols, it has been confirmed that some differences in map reading depend on the legend used.
\end{abstract}

Key words: map legend, map use, think-aloud method.

\section{INTRODUCTION}

Currently, access to geospatial information is becoming increasingly easy, and therefore the map becomes a widely used tool. Furthermore, in many fields of human activity, maps are also essential tools in decision-making processes. Hence, there is a need to study the process of map use thoroughly and to asses the significance of individual map design elements. Wider and more detailed knowledge concerning the process of map use allows for the adjustment of map design to the cognitive abilities of map users, and make the entire process of map use more effective.

P.C. Muehrcke (1978) distinguished several "levels" of map use, characterised by an increasing complexity. Firstly, map reading, i.e. reading the basic spatial concepts: position, direction and distance in order to translate the map's features into a mental image of the environment. Secondly, map analysing, i.e. becoming aware of the spatial relations with the assistance 
of cartometrics or pattern comparisons. Thirdly, map interpretation, i.e. recognising patterns and seeking explanations for them. Lastly, orientation, which includes position and route finding. As we can see, the map is a tool for many various tasks and operations.

Cartographers were extensively involved in cognitive map design research during the second part of the $20^{\text {th }}$ century, but they concentrated on simple map reading processes, i.e. estimating the size of the basic graphic variables or determining the smallest noticeable differences (Montello, 2002). Only starting from the 1980s did they realise that a map should be treated as a tool for more complex activities, and map design research should include many variables and various operations.

One of the basic elements of almost every map is the map legend. In many languages, the term "map legend" is translated into the word "key", e.g. "Zeichenschlüssel" in German or "key symbol" in English, etc. In fact, the legend is the key for the understanding of a map and it is essential for the proper interpretation of the map content. The language used within a map differs from the natural language as it introduces a system of signs and provides spatial information. Hence, there is a need for a translation between those languages. The map legend itself is a kind of translator. Moreover, the map legend serves a wide range of functions (Schlichtmann, 1997). It can help the map user to understand the presented themes, their hierarchy and rules of their classification. Map legends can also indicate the way of map reading and help unravel "hidden" information. Due to a direct connection between the structure of information and the design of the legend, the map user, by reading only the legend, can develop some significant conclusions concerning the spatial pattern of the presented objects. This makes the legend a very valuable element of the map, which provides the basis for knowledge schemes used to understand maps.

Unfortunately, the importance of the map key has been undervalued so far. In 1967, V. Geabler worried about neglecting the map legend by cartographers; 30 years later, the situation did not change: H. Schlichtmann (1997) also complained that the map legend deserved more attention that it had received. Only a small number of controlled experiments concerning the map legend has been conducted (e.g. DeLucia, Hiller, 1982; Pickle et al., 1995; Kraak et al., 1997), and therefore we can say that the role of map legends in the process of map use has not been studied sufficiently. Furthermore, in the era of GIS in cartography and sciences related to spatial information, many maps are produced by non-cartographers. An analyses of selected GIS software packages (Dmochowski, Pasławski, 2000; Bajer, Korycka-Skorupa, 2008) indicated that map design related to the map legend seems to be undervalued in this software: only a limited scope of legend constructions is available, many of them with poor graphic design. Hence, it seems worthwhile to determine how the choice of the map legend construction may affect the process of map use. A good way to achieve this goal is to monitor the work of map users while executing these tasks. This kind of exploratory 
study seems to be a helpful source of qualitative and quantitative knowledge concerning the map use process.

\section{THE EXPERIMENT}

In order to answer some selected questions about the role of the map legend in the map use process, a controlled experiment was conducted. Based on the provided maps, the participants were supposed to determine the best locations for 3 objects with different location requirements: a dumping site, a luxury hotel and a plantation. The experiment aimed to simulate the execution of problem-solving tasks based on the information derived from maps with differently designed legends, whereas the map face would continue to be the same. A map with several thematic layers was used (Fig. 1), viz.: settlements and related objects, forests, roads, traffic volume, nature conservation, soil permeability and profitability of farming subsided crops.

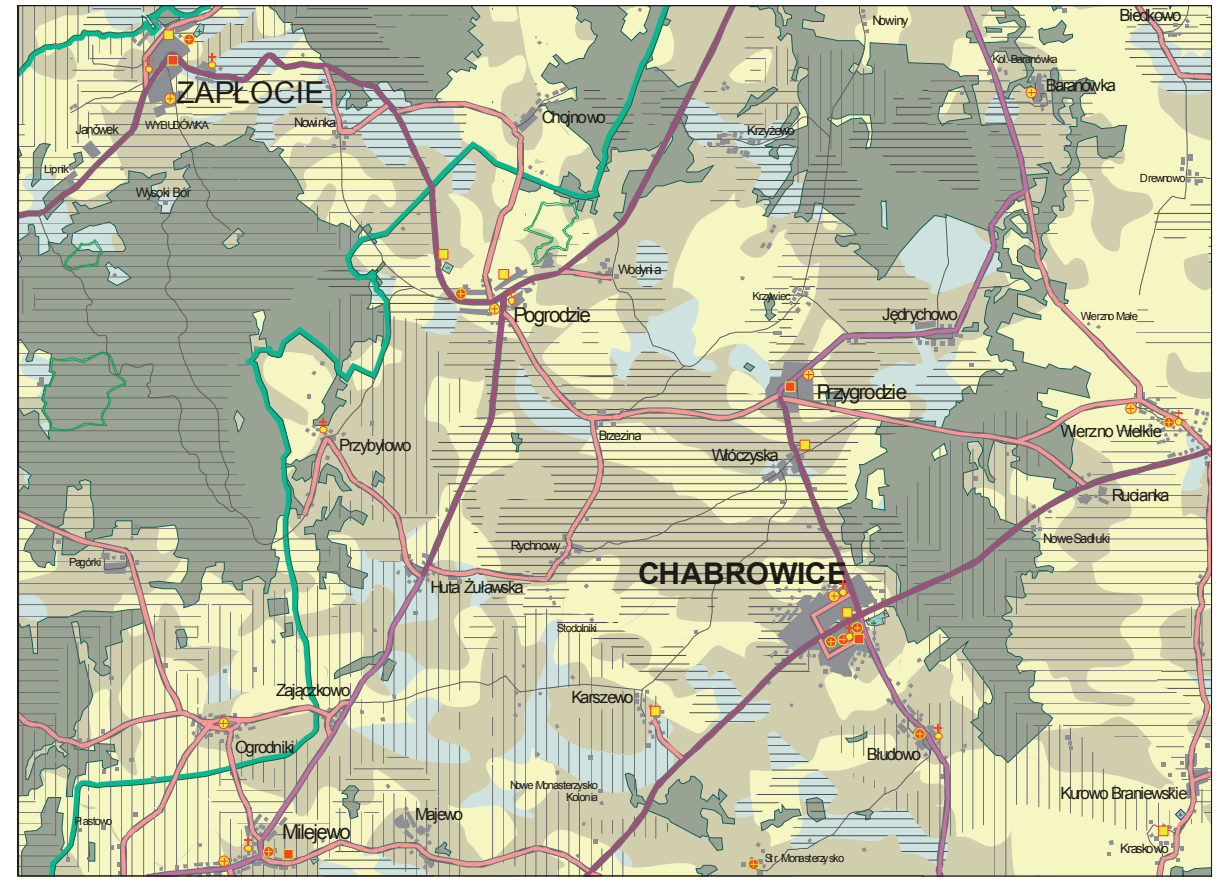

Fig. 1. Thematic map used for problem-solving tasks (scale reduced).

Maps contained information on nominal and ordinal measurement level. The signs used were designed in compliance with the cartographic conventions (e.g. forests are green), but as far as possible the signs were not 
intuitive, which made the legend an essential feature for the proper understanding of the map content.

Three differently designed legends were prepared: list-legend (Fig. 2A), grouping-legend (Fig. 2B) and natural-legend (Fig. 2C).

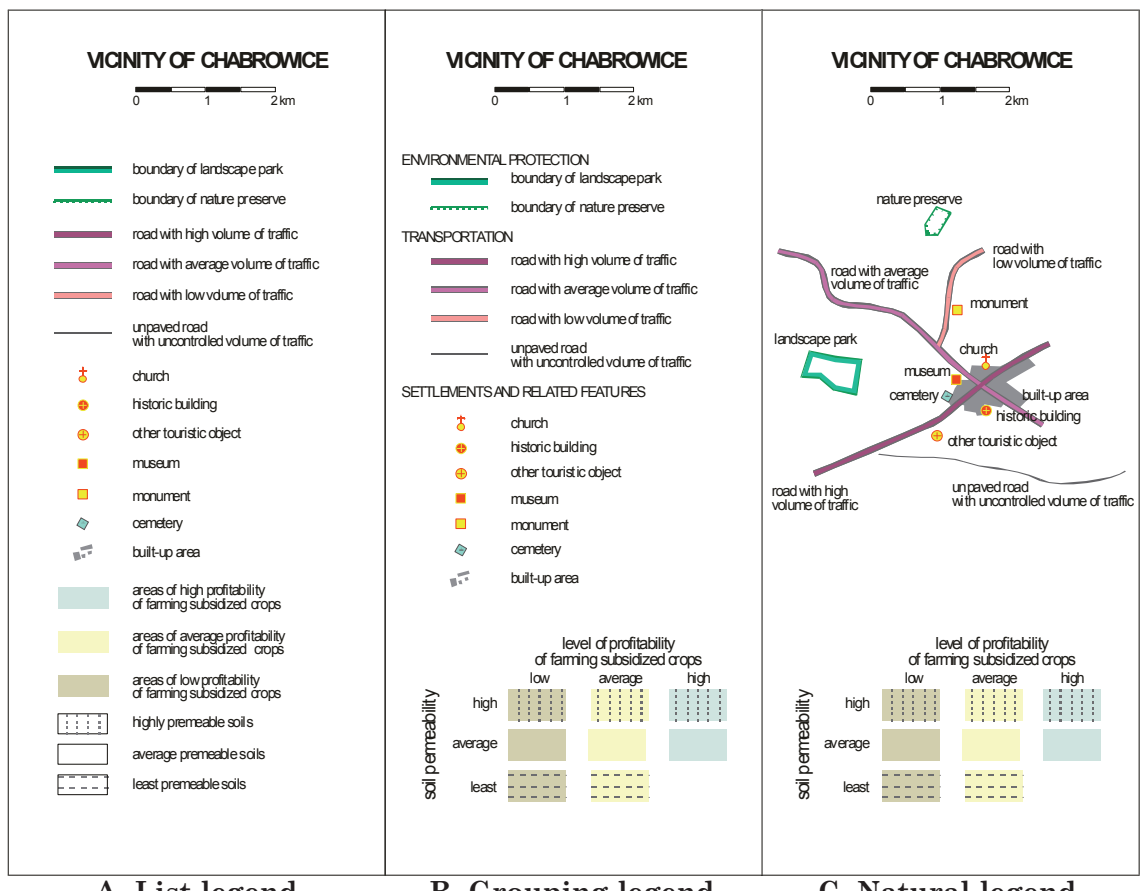

A. List-legend

B. Grouping-legend

C. Natural-legend

Fig. 2. Differently designed legends applied in the experiment (scale reduced).

All the constructions used are considered by cartographers as proper ones and are often used in cartographic studies. The first construction is the list-legend, with the order of specimens that is often used on e.g. on tourist maps, i.e. according to the shape of the signs: first line symbols, then point, and area one at the end. The second, grouping-legend, was constructed by grouping the specimens into four general semantic categories. Furthermore, the area symbols were arranged as a matrix legend. The third construction, natural-legend, contains the natural legend and the same matrix legend as in the grouping-legend.

It was valuable to monitor not only the results (i.e. only the answer) of the tasks, but also the whole process of map use, the process of knowledge acquisition from the map, as well as the applied problem-solving strategy. To meet all the requirements, the think-aloud method was applied in the experiment, which meant that the participants were asked to voice their thoughts during the exercise. The thinking aloud was recorded, transcribed and coded into verbal protocols containing the source of direct and in-depth 

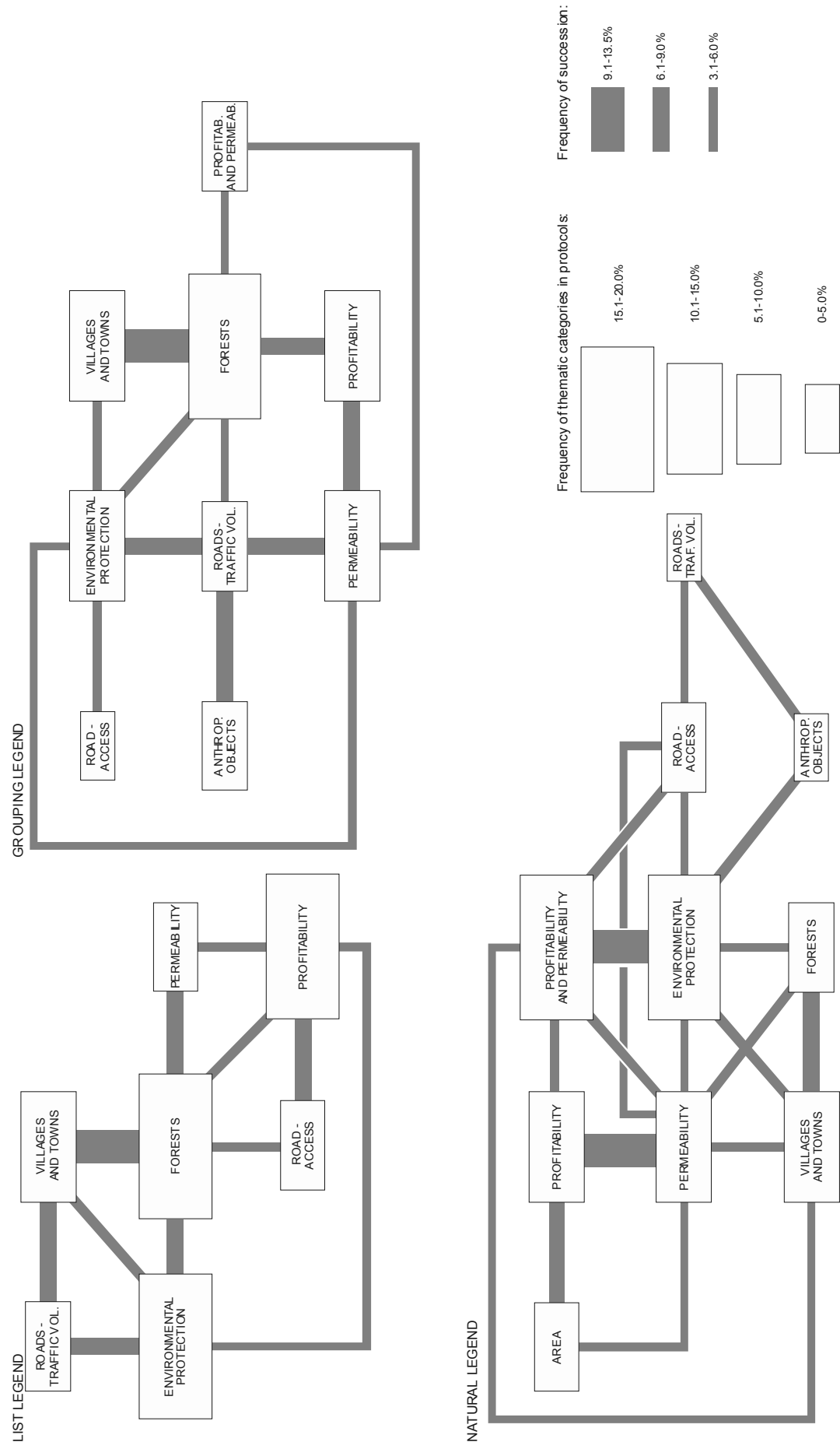

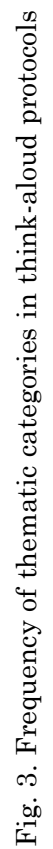

焉 
qualitative information on cognitive processes. Not only was the result of task important, but also the whole process. Hence, while analysing the verbal protocols, the ways of using information derived from the legends received a lot of attention.

Twelve participants took part in the experiment. All of them were graduate students (fourth semester of Territorial Management studies at the Faculty of Geography and Regional Studies, University of Warsaw).

\section{RESULTS}

Based on the coded think-aloud protocols, three graph were prepared (Fig. 3 ). The graphs show how often the participants used specific thematic categories and in what succession.

It turned out that the list-legend produces clear patterns of map reading. The succession of categories is connected with the order of specimens in the legend. Exceptions from the order indicated by the legend may be the result of skipping some of the signs, and reading the symbols listed below. The graph also shows that the list-legend results in the smallest number of thematic categories used. It can be explained by the fact that the participants facing such a long row of signs felt overwhelmed by the information and behaved like "cognitive misers": they did not need to perceive all the available information to make the decision, but only the scope that they considered to be sufficient. That kind of behaviour is connected with the limited capacity of human working memory.

A different graph was developed on the basis of the protocols of those participants who used the natural-legend. The pattern of map reading is not clear and the succession was not repetitive among the participants (numerous, but not strong connections between the thematic categories could be observed). The construction does not indicate the order of map reading. The natural legend does not provide any further information about the thematic groups: their extent or number of objects in each thematic layer. On the other hand, the natural-legend results in the greatest number of categories used. Visually, this construction is the most compact one, so it is the easiest to glance at it among all the applied legend constructions.

Also, a difference could be seen in the interpretation of signs depending on whether the specimens were put in matrix legend or in the list (e.g. grouping- vs. list-legend). When using the matrix legend, most users treat both kinds of information as a whole, regardless of whether one piece of information was useless or not. As a result, only the list-legend graph does not contain the category: "Profitability and permeability", the two characteristics that were only analysed separately by the participants using the list-legend. The participants who used the grouping- and the natural-legends (both containing the matrix legend) analysed the two characteristics at the same time. The set of these two characteristics was most often analysed by 
the participants who used the natural-legend. Actually, the information about profitability and permeability was the most easily available, because it was put in the matrix legend. The rest of the information was not ordered according to semantic criteria, and therefore it was harder to acquire.

Think-aloud protocols were also coded based on the nature of the operation during the problem-solving stage. It was revealed that there is no clear relationship between the legend construction and the strategy applied. The participants using the same legend construction used different strategies. The type of strategy applied is more strongly connected with previous education and training in map reading. The users who had problems with understanding the map content and executing even simple tasks (worse solvers) rarely used the legend. They often guessed the meaning of signs, and more frequently used the information that does not require its meaning to be verified, As a consequence, they skipped even important information and focused on information presented using intuitive signs. Even when feeling confused, they did not treat the legend as a helpful feature. On the other hand, the users who easily absorbed the information and successfully executed other tasks (better solvers) used the legend very often. They started with reading the legend, and then located each sign on the map face. They seldom guessed the meaning of the sign; even the meaning of the intuitive signs (e.g. forests) was verified with the legend content. To sum up, there are various strategies of problem solving, but they do not seem to be significantly connected with the legend construction.

The number of participants in the experiment makes it impossible to apply sophisticated statistical measures. The results discussed above show the direction for further explorations and studies. This kind of research is going to be continued in order to collect sufficient data to provide an answer to the question whether there are any statistically significant relations between map legend design and the ways of map use.

\section{REFERENCES}

Bajer A., Korycka-Skorupa J., 2008, Kartodiagram w wybranych programach komputerowych [Diagram maps in selected computer software], Polski Przeglad Kartograficzny, Vol. 40, No. 3, 247-266.

Dmochowski M., Pasławski J., 2000, Kartogram w wybranych programach komputerowych [Choropleth maps in selected computer software], Polski Przeglad Kartograficzny, Vol. 32 , No. 4, 288-301.

Montello D.R., 2002, Cognitive map-design research in the twentieth century: theoretical and empirical approaches, Cartography and Geographic Information Science, Vol. 29, No. 3, 283-304.

Gaebler V., 1967, Die Legende thematischer Karten. Batrachtung zur Gestaltung, Vermessungstechnik, Vol. 15, 304-309.

Muehrcke P.C., 1978, Map use. Reading, analysis, and interpretation, JP Publication, Madison. Schlichtmann H., 1997, Functions of the map legend, [in:] Proceedings of the 18th International Cartographic Conference, 430, Stockholm. 
DeLucia A.A, Hiller D.W., 1982, Natural legend design for thematic maps, The Cartographic Journal, Vol. 19, No. 1, 46-52.

Pickle L. W., Herrmann D., Wilson B., 1995, A legendary study of statistical map reading: The cognitive effectiveness of statistical map legends. [in:] Pickle, L. W., Herrmann, D. (eds.): Cognitive Aspects of Statistical Mapping, NCHS Working Paper Series Report, No. 18, 233-248. National Center for Health Statistics, Hyattsville.

Kraak M.-J., Edsall R., MacEachren A.M., 1997, Cartographic animation and legend for temporal maps: exploration and/or interaction, [in:] Proceedings of the 18th International Cartographic Conference, 253-260, Stockholm. 